\title{
Acompanhamento presencial e à distância para o tratamento da enurese noturna com alarme ${ }^{1}$
}

\author{
Face-to-face and long-distance protocols \\ in treatment of enuresis by alarm
}

\author{
Rodrigo Fernando PEREIRA ${ }^{2}$ \\ Yasmin Spaolonzi DAIBS ${ }^{2,3}$ \\ Paula Ferreira BRAGA ${ }^{4}$
}

Edwiges Ferreira de Mattos SILVARES ${ }^{4}$

\begin{abstract}
Resumo
A enurese é definida como a micção normal que ocorre durante o sono. Para o seu diagnóstico, são necessárias a idade mínima de cinco anos e frequência de pelo menos um episódio por mês. As causas mais consensuais são a poliúria noturna, dificuldades em despertar e a hiperatividade detrusora. O objetivo deste trabalho foi comparar crianças e adolescentes enuréticos que se submeteram ao tratamento com alarme, divididas entre dois protocolos de tratamento: presencial e à distância. Participaram do trabalho 61 crianças e adolescentes com idades entre seis e 17 anos e suas famílias. Todos participaram de duas sessões presenciais nas quais foram fornecidas informações sobre a enurese e o tratamento baseado no programa de espectro total. Os participantes foram alocados randomicamente entre protocolos de acompanhamento presencial $(n=27)$ e à distância $(n=34)$. Verificou-se que as crianças e adolescentes acompanhados à distância apresentam resultado comparável ao relatado na literatura, sendo uma alternativa viável ao acompanhamento presencial.
\end{abstract}

Unitermos: Adolescente. Criança. Enurese. Tratamento.

\begin{abstract}
Nocturnal enuresis is defined as natural urination during sleep occurring at least once a month among children aged fiveyears or older. There is a relative consensus in literature regarding possible causes: nocturnal polyuria, difficulties in waking up and a hyperactive bladder. The objective of this work was two compare two treatment protocols which used an alarm to treat enuresis: face-to-face and long distance. Sixty one enuretic children aged between 6 and 17 and their parents or caregivers participated in the study. They all attended two initial face-to-face sessions in which they received information on the treatment based on the full-spectrum programme. Participants were randomly allocated to the face-to-face $(n=27)$ and long-distance $(n=34)$ protocols. The long-distance protocol recorded outcome rates comparable to those cited in literature and thus offered a feasible alternative to the face-to-face protocol.
\end{abstract}

Uniterms: Adolescent. Child. Enuresis. Treatment.

\section{urvt}

1 Artigo elaborado a partir da tese de R. F. PEREIRA, intitulada "Variáveis moderadoras do resultado da intervenção com alarme para a enurese noturna". Universidade de São Paulo, 2010. Apoio: Fundação de Amparo à Pesquisa do Estado de São Paulo.

2 Universidade de São Paulo, Instituto de Psicologia, Departamento de Psicologia Clínica. Av. Prof. Mello Moraes, 1721, Bloco F, Sala 30, Cidade Universitária, 05508-030, São Paulo, SP, Brasil. Correspondência para/Correspondence to: R. F. PEREIRA. E-mail: <rpereira@usp.br>.

3 Universidade Presbiteriana Mackenzie, Centro de Ciências Biológicas e da Saúde. São Paulo, SP, Brasil.

4 Pontifícia Universidade Católica de São Paulo, Instituto de Psicologia, Programa de Psicologia Experimental. São Paulo, SP, Brasil. 
A enurese é definida como a micção normal que ocorre durante o sono em crianças com ao menos cinco anos de idade. Em relação à frequência de ocorrência dos episódios, duas vertentes são atualmente apresentadas na literatura. A primeira, proposta pela International Children Continence Society (ICCS) (Nevéus et al., 2006) estabelece para o diagnóstico a frequência de um episódio por mês, enquanto a segunda, proposta pela American Psychiatric Association - APA (2002), estabelece a ocorrência de dois episódios por semana, por um período de pelo menos três meses.

Com essa discrepância nos critérios, é esperado que os estudos sobre a prevalência sejam discordantes, dependendo da severidade necessária para que se caracterize a enurese. Butler, Golding, Northstone \&The ALSPAC Study Team (2005) verificaram que, aos sete anos e meio de idade, 15,5\% de uma amostra de crianças inglesas relatam molhar a cama, mas apenas 2,6\% diziam fazê-lo numa frequência suficiente para que o critério estabelecido pela APA fosse atingido.

De acordo com Nevéus et al. (2009), o conhecimento científico sobre a enurese noturna avançou nos últimos dois anos. Às três hipóteses etiológicas mais consensuais (Butler \& Holland, 2000) - poliúria noturna por deficit na produção de vasopressina, dificuldades em despertar e hiperatividade detrussora - juntaram-se outros aspectos, como a perspectiva de que o sono das crianças enuréticas pode levar a outros problemas de ordem cognitiva (Nevéus, 2008).

Ainda que tenha havido progressos na compreensão do quadro, não houve mudança em relação aos tipos de tratamento recomendados (Hjälmas et al., 2004). Os únicos que recebem nível de recomendação e evidência A são a desmopressina, análogo sintético da vasopressina e o tratamento psicológico com alarme.

O sistema de tratamento com alarme, criado em 1938 por Mowrer e Mowrer, é um dos mais bem sucedidos procedimentos na história da terapia comportamental, alcançando uma taxa de sucesso, expressa em semanas totalmente secas, na ordem dos 65\%. Tendo sido aperfeiçoado ao longo do tempo, até atingir formatos mais abrangentes como o full spectrum home training, de Houts (2003), a técnica tem potencializado seus resultados, alcançando a diminuição da recaída de $40 \%$ para $10 \%$, através de um procedimento de 184 generalização chamado de superaprendizagem.
O alarme consiste em um sensor que é colocado na cama ou na roupa da criança, ligado a um dispositivo que emite um som quando ocorre um episódio de enurese. Apesar de sua efetividade, ainda não se tem certeza sobre porque o alarme funciona. Uma lista de possíveis causas para os resultados desse tratamento incluem alterações sociais e motivacionais, condicionamento por esquiva ativa, resposta muscular condicionada, aumento na capacidade funcional e diminuição na produção noturna de urina (Butler et al., 2007), sendo que os autores enfatizam a última possibilidade, por verificar que crianças tratadas com o alarme têm um aumento na concentração da urina.

Uma vez que o mecanismo de funcionamento do alarme ainda não está totalmente claro, e tendo em vista que pelo menos 30\% das crianças que se submetem a ele não obtêm sucesso, pode-se perguntar se o papel do terapeuta comportamental no tratamento da enurese contribui para o alcance da taxa de sucesso.

Grande parte da literatura afirma que os acompanhamentos para o uso do alarme são realizados através de contatos telefônicos, em que são investigados pontos-chave do procedimento a ser realizado em casa pelas famílias. Apenas um trabalho (Houts, 2003) cita brevemente uma comparação entre o acompanhamento do alarme com duas visitas e múltiplas visitas ao clínico, alegando não haver diferença nos resultados, mas sem apresentar dados concretos. Nevéus et al. (2010) comenta ainda que são necessários poucos contatos telefônicos de acompanhamento após a inserção do alarme, mas também sem dados objetivos. Desta forma, o presente trabalho teve como objetivo comparar crianças e adolescentes enuréticos que se submeteram ao tratamento com alarme, divididas entre dois protocolos de tratamento: presencial e à distância. Como objetivos secundários, verificou-se a relação entre os resultados dos tratamentos e índice de problemas de comportamento, escolaridade dos pais, sexo, idade e frequência de molhadas na linha de base.

Hipotetizou-se que o acompanhamento presencial, mais frequente e próximo, traria ganhos às famílias, expressos em maior eficácia e rapidez na obtenção do controle das noites secas. Por outro lado, é possível esperar que o número de desistências no acompanhamento presencial seja maior, pelas dificuldades das famílias em comparecer à clínica. Foram também formuladas hipóteses de que baixo nível de escolaridade 
dos pais, alta frequência inicial de episódios e alto índice de problemas de comportamento prejudicariam o resultado do tratamento, independentemente do tipo de acompanhamento realizado. Acreditamos que entender melhor a influência da forma de acompanhamento no resultado do tratamento para a enurese possa ajudar a elaborar formas mais eficientes de trabalhar com a enurese em contextos institucionais.

\section{Método}

\section{Participantes}

Participaram do trabalho 61 crianças e adolescentes com idades entre seis e 17 anos e suas famílias. A idade média foi igual a 9,47 (DP=2,55), sendo a amostra composta por 44 meninos e 17 meninas. A amostra parece refletir dados epidemiológicos da enurese, uma vez que ela é mais comum no sexo masculino e tende a diminuir com a idade.

O projeto foi aprovado pelo Comitê de Ética em Pesquisa com seres humanos do Instituto de Psicologia da Universidade de São Paulo ofício 6306/ CEPH-20/12/06, em reunião de 04/12/2006. Todos os participantes assinaram um Termo de Consentimento Livre e esclarecido antes de sua inclusão na amostra.

\section{Instrumentos}

Os problemas de comportamento foram avaliados através do Inventário de Comportamentos da Infância e da Adolescência (Child Behavior Checklist CBCL) (Achenbach \& Rescorla, 2001), antes do início do tratamento. $O$ instrumento foi selecionado por fornecer escores de problemas internalizantes, externalizantes e totais, permitindo uma análise mais detalhada da influência de características comportamentais no resultado do tratamento.

Cada família recebeu também folhas de registro padronizadas, em que deveria anotar, diariamente, se houve ou não episódio de enurese, a hora em que ocorreu, a quantidade aproximada de urina, e se a criança acordou ou não com o som do alarme.

\section{Procedimentos}

Todos os participantes, juntamente com seus pais ou cuidadores, participaram de duas sessões presenciais, nas quais foram fornecidas informações sobre a enurese e o tratamento baseado no programa de espectro total descrito por Houts (2003). Os materiais fornecidos foram folhas para o registro de episódios, um guia com as instruções (Houts, 1990) e os alarmes empregados no tratamento, ao término da segunda sessão. Os participantes foram instruídos ao uso correto do alarme, bem como ao preenchimento das folhas de registro.

Imediatamente após as duas sessões presenciais, os participantes foram alocados randomicamente entre protocolos de acompanhamento presencial $(n=27)$ e à distância ( $n=34)$. Os grupos foram testados para verificação de igualdade das seguintes variáveis: sexo, idade média, nível de educação dos pais, médias de escores de problemas de comportamento e frequência inicial de episódios, sem que houvesse discrepância. $\mathrm{O}$ acompanhamento presencial foi caracterizado por sessões semanais no centro de atendimento psicológico do Instituto de Psicologia da Universidade de São Paulo. Já o acompanhamento à distância deu-se através de contatos telefônicos a cada duas semanas. Ambos os protocolos incluíram, no momento dos contatos: a) questionamento sobre os episódios de molhadas (ocorrência, hora e tamanho das manchas de urina); b) questionamento sobre se a criança acordava ou não com o alarme; c) informações sobre o tratamento e os seus procedimentos e d) reasseguramento e novas orientações para pais e crianças conforme necessário (Anexo).

O mesmo terapeuta conduziu as sessões iniciais e os acompanhamentos. Um arquivo eletrônico individual para cada participante era atualizado a cada contato feito com a família. Duas juízas verificaram o conteúdo das sessões a partir de gravações em vídeo, concluindo que todos os procedimentos foram explicados aos grupos que receberam ambos os tipos de atendimento.

O tratamento levou até 28 semanas. Para avaliar os resultados, foi utilizado o critério mais comum na literatura (Butler, Robinson, Holland \& Doherty-Williams, 2004): a) sucesso: pelo menos 14 noites consecutivas sem episódios, dentro do período de tratamento; b) insucesso: menos de 13 noites consecutivas sem episódios, dentro do período de tratamento e c) desistência: não comparecimento às sessões presenciais ou não seguimento dos procedimentos (por exemplo o uso do alarme) em casa. 


\section{Resultados}

Trinta e sete participantes, compondo 60\% da amostra, obtiveram sucesso, ou seja, pelo menos 14 noites secas consecutivas dentro do período de 28 semanas do tratamento com alarme. O número de insucessos e desistências foi idêntico: 12, o que equivale a 20\% da amostra (Tabela 1).

Como pode ser observado, ambos os grupos são iguais em relação à idade. Embora a média de idade seja quase um ano maior no grupo atendido à distância, essa variação não foi suficiente para configurar uma diferença significativa. Outra análise possível, subdividindo os participantes entre crianças ( 6 a 10 anos) e adolescentes (11 a 17 anos), mostra que a porcentagem de ambas as faixas etárias em cada grupo é praticamente idêntica.

Há predominância do sexo masculino na amostra como um todo, o que se mantém entre os grupos divididos pelo tipo de acompanhamento, ainda que a porcentagem de meninos atendidos à distância seja maior do que os atendidos presencialmente.

O mesmo ocorre em relação à escolaridade dos pais: há uma predominância daqueles que têm o terceiro grau sobre os que têm níveis inferiores de formação, sendo que essa ocorrência é similar nos dois grupos de acompanhamento.
Na Tabela 2, constam os valores médios das somas de escalas do CBCL, divididos de acordo com os grupos, bem como o número de crianças cujo escore se encontra num patamar clínico (acima de 60) e aquelas cujo escore se encontra abaixo dessa faixa.

O que pode ser observado é que, em relação às médias dos grupos, não há diferenças estatisticamente significativas para nenhum dos três escores, o que indica que os grupos eram semelhantes nesse aspecto. No entanto, verifica-se que havia maior número de participantes com escores de problemas totais na faixa clínica entre os acompanhados presencialmente (Figura 1).

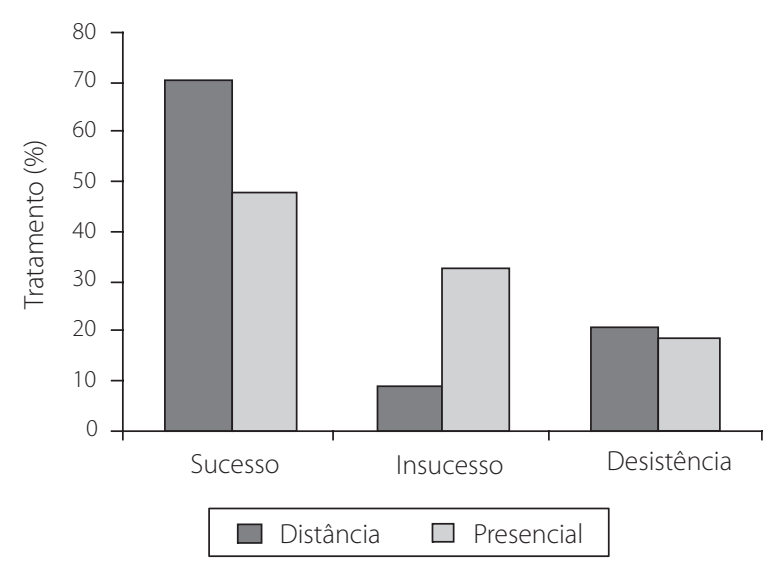

Figura1. Resultado do tratamento de acordo com o tipo de atendimento.

Tabela 1.Comparação de dados iniciais de acordo com o tipo de acompanhamento. São Paulo (SP), 2007-2010.

\begin{tabular}{|c|c|c|c|c|c|c|c|c|c|c|c|c|c|}
\hline \multirow{2}{*}{ Variáveis } & \multicolumn{4}{|c|}{ Geral } & \multicolumn{4}{|c|}{ Distância } & \multicolumn{4}{|c|}{ Presencial } & \multirow{2}{*}{ Valor - $p$} \\
\hline & $n$ & $\%$ & M & DP & $n$ & $\%$ & $M$ & DP & $\mathrm{n}$ & $\%$ & M & DP & \\
\hline Pacientes & 61 & 100 & & & 34 & 55,7 & & & 27 & 44,3 & & & \\
\hline \multirow{2}{*}{ Idade (anos) } & & & & & & & & & & & & & Teste igualdade de média \\
\hline & & & 9,5 & 2,57 & & & 9,9 & 2,83 & & & 9,0 & 2,17 & 0,201 \\
\hline \multicolumn{14}{|l|}{ Sexo } \\
\hline Frequência & & & & & & & & & & & & & Teste de independência \\
\hline Feminino & 17 & 27,9 & & & 7 & 20,6 & & & 10 & 37 & & & 0,250 \\
\hline Masculino & 44 & 72,1 & & & 2 & 79,4 & & & 17 & 63 & & & \\
\hline \multicolumn{14}{|c|}{ Escolaridade dos pais } \\
\hline Frequência & & & & & & & & & & & & & Teste de independência \\
\hline $10 \mathrm{grau}$ & 5 & 8,9 & & & 3 & 9,4 & & & 2 & 8,3 & & & 0,608 \\
\hline $2^{\circ} \mathrm{grau}$ & 18 & 32,1 & & & 12 & 37,5 & & & 6 & 25 & & & \\
\hline $3^{\circ} \mathrm{grau}$ & 33 & 58,9 & & & 17 & 53,1 & & & 16 & 66,7 & & & \\
\hline \multicolumn{14}{|c|}{ Frequência de molhadas } \\
\hline Linha de base (em & & & & & & & & & & & & & Teste Mann Whitey \\
\hline 2 semanas) & & & 10,5 & 4,33 & & & 10,1 & 4,41 & & & 11,0 & 4,24 & 0,308 \\
\hline
\end{tabular}

M: Média; DP: Desvio-Padrão. 
Pode-se perceber que o número relativo de sucessos foi maior no grupo atendido à distância, que também obteve menor número relativo de insucessos, porém número de desistências ligeiramente maior. A partir desses valores, obtém-se uma taxa de sucesso de $48 \%$ no acompanhamento presencial e $71 \%$ no acom- panhamento à distância. A diferença entre os grupos foi significativa $(p=0,022)$ (Tabela 3$)$.

Em relação à idade média, não há diferença entre os participantes que obtiveram sucesso ou insucesso, sendo próxima a 10 anos de idade em ambos os casos. O mesmo acontece quando a amostra é dividida nas

Tabela 2. Comparação dos níveis de problemas de comportamento de acordo com o tipo de acompanhamento. São Paulo (SP), $2007-2010$.

\begin{tabular}{|c|c|c|c|c|c|c|c|c|c|}
\hline \multirow{2}{*}{ Variáveis $\quad$ Acompanhamento } & \multicolumn{4}{|c|}{ Distância } & \multicolumn{4}{|c|}{ Presencial } & \multirow{2}{*}{ Valor - $p$} \\
\hline & $n$ & $\%$ & $M$ & $\mathrm{DP}$ & $n$ & $\%$ & M & DP & \\
\hline Pacientes $(n=61)$ & 34 & 55,7 & & & 27 & 44,3 & & & \\
\hline \multirow[t]{3}{*}{ Internalizantes } & & & & & & & & & Teste igualdade de média \\
\hline & & & 62,9 & 7,13 & & & 61,7 & 7,65 & 0,524 \\
\hline & & & & & & & & & Teste de independência \\
\hline Não clínico (<60pontos) & 13 & 38,2 & & & 9 & 33,3 & & & 0,791 \\
\hline Clínico ( $>$ ou =60 pontos) & 21 & 61,8 & & & 18 & 66,7 & & & \\
\hline \multirow[t]{3}{*}{ Externalizantes } & & & & & & & & & Teste igualdade de média \\
\hline & & & 58,8 & 10,75 & & & 61,2 & 8,95 & 0,363 \\
\hline & & & & & & & & & Teste de independência \\
\hline Não clínico (<60 pontos) & 17 & 50 & & & 12 & 44,4 & & & 0,797 \\
\hline Clínico (> ou =60 pontos) & 17 & 50 & & & 15 & 55,6 & & & \\
\hline \multirow[t]{3}{*}{ Total } & & & & & & & & & Teste igualdade de média \\
\hline & & & 63,0 & 9,51 & & & 64,1 & 7,32 & 0,598 \\
\hline & & & & & & & & & Teste de independência \\
\hline Não clínico (<60 pontos) & 16 & 47,1 & & & 5 & 18,5 & & & 0,03 \\
\hline Clínico (> ou =60 pontos) & 18 & 52,9 & & & 22 & 81,5 & & & \\
\hline
\end{tabular}

M: Média; DP: Desvio-Padrão.

Tabela 3. Comparação entre participantes que obtiveram sucesso ou não no tratamento em relação a possíveis variáveis moderadoras. São Paulo (SP), 2007-2010.

\begin{tabular}{|c|c|c|c|c|c|c|c|c|c|c|c|c|}
\hline \multirow{2}{*}{ Perfil sociodemográfico } & \multicolumn{4}{|c|}{ Geral } & \multicolumn{4}{|c|}{ Insucesso } & \multicolumn{3}{|c|}{ Sucesso } & \multirow{2}{*}{ Valor - $p$} \\
\hline & $n$ & $\%$ & $M$ & $\mathrm{DP}$ & $n$ & $\%$ & $\mathrm{M}$ & $\mathrm{DP}$ & $\mathrm{n}$ & $\%$ & M DP & \\
\hline Pacientes & $61^{*}$ & 100,0 & & & 12 & 24,5 & & & 37 & 75,5 & & \\
\hline \multirow[t]{3}{*}{ Idade (anos) } & & & & & & & & & & & & Teste igualdade de média \\
\hline & & & 9,5 & 2,57 & & & 9,7 & 2,10 & & & $9,9 \quad 2,83$ & 0,778 \\
\hline & & & & & & & & & & & & Teste de independência \\
\hline Criança (06 a 10 anos) & 41 & 67,2 & & & 7 & 58,3 & & & 22 & 59,5 & & 1,000 \\
\hline Adolescente (11 a 17 anos) & 20 & 32,8 & & & 5 & 41,7 & & & 15 & 40,5 & & \\
\hline Total & 61 & 100,0 & & & 12 & 100,0 & & & 37 & 100,0 & & \\
\hline Sexo & & & & & & & & & & & & Teste de independência \\
\hline Jexu & & & & & & & & & & & & 0,466 \\
\hline Feminino & 17 & 27,9 & & & 2 & 16,7 & & & 12 & 32,4 & & \\
\hline Masculino & 44 & 72,1 & & & 10 & 83,3 & & & 25 & 67,6 & & \\
\hline Escolaridade pais & & & & & & & & & & & & Teste de independência \\
\hline $1^{\circ} \mathrm{grau}$ & 5 & 8,9 & & & 1 & 10,0 & & & 2 & 5,4 & & 0,738 \\
\hline $2^{\circ}$ grau & 18 & 32,1 & & & 3 & 30,0 & & & 12 & 32,4 & & \\
\hline $3^{\circ}$ grau & 33 & 58,9 & & & 6 & 60,0 & & & 23 & 62,2 & & \\
\hline
\end{tabular}

M: Média; DP: Desvio-Padrão; * O número total de participantes inclui também os desistentes, que não foram considerados na análise de significância estatística entre os grupos de sucesso e insucesso. 
categorias criança (6 a 10 anos) e adolescente (11 a 17 anos). A proporção de sucessos e insucessos é praticamente idêntica em ambas as faixas etárias.

O sexo também não foi um fator determinante para obtenção do sucesso nessa amostra. Embora apenas 2 meninas não tenham atingido o critério de sucesso, contra 10 meninos, enquanto a proporção de sucesso foi de 12 para 25, não se configurou uma diferença estatisticamente significativa (Tabela 4).

Outra variável que não influenciou o resultado foi a escolaridade dos pais, dado que o número de sucessos e insucessos distribuiu-se de forma semelhante entre os três níveis apresentados.

A comparação entre os participantes que obtiveram sucesso ou não no tratamento, em relação a problemas de comportamento, foi realizada com base na média dos escores obtidos e na proporção de participantes com escores clínicos para cada soma de escalas do CBCL.

Verifica-se que os participantes, bem sucedidos ou não, tiveram escores semelhantes de problemas internalizantes, com média acima da faixa. A proporção de participantes com escore clínico também foi semelhante entre os grupos com os dois tipos de resultado.
Já a média dos escores de problemas externalizantes apresentou uma diferença estatisticamente significativa. Os participantes que não obtiveram sucesso no tratamento apresentaram escore médio na faixa clínica e mais alto do que os bem-sucedidos, cujo escore médio situou-se abaixo daquela faixa. Uma tendência no mesmo sentido pode ser encontrada na distribuição dos participantes com escores clínicos nos dois grupos.

Ainda assim, quando se considera o escore de problemas totais, pode-se observar uma tendência de os participantes que não obtiveram sucesso apresentarem uma média mais alta neste escore, sendo, portanto, mais clínicos que os participantes bem-sucedidos. De fato, em termos de frequência, os participantes dos dois grupos estão distribuídos de maneira estatisticamente diferente entre os dois perfis.

\section{Discussão}

De acordo com os resultados descritos anteriormente, as crianças atendidas presencialmente apresentaram duas características que, embora não significantes estatisticamente, diferenciam daquelas atendidas à

Tabela 4. Comparação dos escores iniciais de problemas de comportamento de acordo com o resultado do tratamento. São Paulo (SP), 20072010.

\begin{tabular}{|c|c|c|c|c|c|c|c|c|c|}
\hline \multirow{3}{*}{ Variável } & \multicolumn{8}{|c|}{$\mathrm{CBCL}$} & \multirow{3}{*}{$\begin{array}{c}\text { Comparação/Estatística } \\
\text { Entre sucesso e insucesso } \\
\text { valor - } p\end{array}$} \\
\hline & \multicolumn{4}{|c|}{ Insucesso } & \multicolumn{4}{|c|}{ Sucesso } & \\
\hline & $\mathrm{n}$ & $\%$ & M & $\mathrm{DP}$ & $\mathrm{n}$ & $\%$ & $M$ & DP & \\
\hline Pacientes ${ }^{*}$ & 12 & 24,5 & & & 37,0 & 75,5 & & & \\
\hline \multirow[t]{3}{*}{ Internalizantes } & & & & & & & & & Teste igualdade de média \\
\hline & & & 63,9 & 6,30 & & & 62,0 & 8,15 & 0,467 \\
\hline & & & & & & & & & Teste de independência \\
\hline Não clínico (<60ptos) & 3 & 25,0 & & & 14,0 & 37,8 & & & 0,503 \\
\hline Clínico (> ou =60ptos) & 9 & 75,0 & & & 16,0 & 43,2 & & & \\
\hline \multirow[t]{3}{*}{ Externalizantes } & & & & & & & & & Teste igualdade de média \\
\hline & & & 65,7 & 7,83 & & & 57,1 & 8,73 & 0,004 \\
\hline & & & & & & & & & Teste de independência \\
\hline Não clínico (<60ptos) & 3 & 25,0 & & & 56,8 & 21,0 & & & 0,095 \\
\hline Clínico (> ou =60ptos) & 9 & 75,0 & & & 43,2 & 16,0 & & & \\
\hline \multirow[t]{3}{*}{ Total } & & & & & & & & & \\
\hline & & & & & & & & & Teste igualdade de média \\
\hline & & & 66,8 & 6,72 & & & 62,2 & 8,78 & 0,074 \\
\hline Não clínico (<60ptos) & 1 & 8,3 & & & 16,0 & 43,2 & & & Teste de independência \\
\hline Clínico (> ou =60ptos) & 11 & 91,7 & & & 21,0 & 56,8 & & & 0,037 \\
\hline
\end{tabular}


distância. A primeira delas é a superioridade da média do número inicial de episódios no período de 14 dias anteriores ao tratamento, e a segunda a superioridade da média dos problemas externalizantes. Estas duas características são descritas na literatura como preditoras do resultado do tratamento da enurese.

Observa-se também que as médias dos escores totais de problemas de comportamento dos dois grupos são altas, situando-se para ambos dentro da faixa clínica. Embora essas médias sejam semelhantes, a proporção de participantes com escore clínico no grupo atendido à distância é significativamente menor do que a proporção de participantes com escore clínico no grupo atendido presencialmente. Ou seja, entre as crianças atendidas à distância, pouco mais da metade tinha escores clínicos no agrupamento total de problemas de comportamento, enquanto esse índice superava os $80 \%$ entre as crianças atendidas presencialmente.

Esse é um problema metodológico considerável. Nesse sentido Houts (2003) afirma que as crianças com maiores índices de problemas de comportamento apresentam pior resultado no tratamento com alarme, o que foi confirmado por Arantes (2007). Desta forma, a comparação entre os acompanhamentos presencial e grupal fica prejudicada, devendo-se considerar esse aspecto na leitura dos dados subsequentes. Entretanto, ainda assim é possível verificar se o acompanhamento à distância é uma alternativa factível para o tratamento com alarme para enurese.

Contrariando a hipótese inicial de que o acompanhamento presencial seria mais efetivo do que o acompanhamento à distância, as crianças que foram acompanhadas por telefone obtiveram maior número de sucessos iniciais e menor número de insucessos. No entanto, ao se comparars essa taxa com o reportado na literatura (Butler, 2004), verifica-se que o acompanhamento à distância obteve um resultado dentro do esperado para o tratamento com alarme, enquanto o grupo acompanhado presencialmente obteve um número de sucessos aquém do normal. A hipótese mais plausível para explicar essa discrepância é a do maior número de participantes com escore clínico no grupo presencial, o que, conforme comentado anteriormente, leva a uma menor efetividade do tratamento, segundo Houts (2003).

De outro lado, uma série de estudos anteriores, realizados por pesquisadores do próprio Projeto Enurese, indica a eficácia, dentro dos níveis convencionais, do tratamento com alarme associado ao acompanhamento presencial, seja ele realizado individualmente ou em grupo (Silva, 2004; Costa, 2005; Pereira, 2006). Partindo destes achados, supõe-se que seja provável que o tipo de acompanhamento não interfira no resultado do tratamento com alarme.

Dentre as variáveis abordadas neste trabalho, os escores clínicos nas escalas externalizantes e totais do $\mathrm{CBCL}$ parecem ser as únicas que se configuram como moderadoras do resultado do tratamento com alarme, já que alteram as chances de obtenção de sucesso. Pode-se até, com relativa segurança, concluir que, quando uma criança ou adolescente não apresenta escore clínico nessas escalas, o tratamento com alarme pode ser administrado presencialmente ou à distância independentemente de sexo, idade, frequência inicial de molhadas ou escolaridade dos pais, sendo obtido o resultado esperado (entre 60\% e 70\% de chances de sucesso).

No entanto, a presença de problemas de comportamentos externalizantes é de fato uma ameaça ao sucesso, uma vez que o que caracteriza esse tipo de problema é o não seguimento de regras, associado a comportamentos opositores e disruptivos. Sabe-se que o uso do alarme depende do seguimento de regras, já que o tratamento implica uma série de mudanças comportamentais tanto dos pais como da criança. Sendo assim, parece clara a relação entre o escore externalizantes na faixa clínica e o insucesso.

Houts (2003) sugere que esses problemas sejam tratados antes de se abordar a enurese terapeuticamente. De fato, a administração de um tratamento que envolva a mudança numa série de contingências e pode gerar conflitos, especialmente para pais intolerantes, não deve ocorrer quando as chances de sucesso serão reduzidas. Isso iria contra a própria função da atuação terapêutica, que é a diminuição do sofrimento e o aumento de contingências reforçadoras (Meyer \& Vermes, 2001).

É questionável, então, se a enurese deve ser deixada em segundo plano nesses casos, ou se deve ser tentado um tratamento que foque tanto a enurese quanto os problemas de comportamento. O procedimento a ser adotado dependerá das condições do serviço no qual o tratamento é oferecido. Contudo, pode-se ressaltar que uma ampla a avaliação psicológica nos casos de enurese é tão importante quanto a avaliação médica, 
pois pode revelar situacões em que o tratamento com alarme não é indicado, ao menos enquanto os problemas externalizantes não forem abordados.

\section{Considerações Finais}

O principal objetivo deste trabalho foi comparar dois tipos de acompanhamento do tratamento com alarme para a enurese. Foi verificado que as crianças e adolescentes acompanhados à distância apresentaram, nesta amostra, melhor resultado do que aquelas atendidas presencialmente. Observou-se também que as crianças com índices mais altos de problemas de comportamento tiveram pior resultado do que aquelas com índices fora da faixa clínica. No entanto, como essas duas variáveis se sobrepuseram na composição dos grupos, é impossível dizer, com certeza, se ambas são determinantes ou apenas uma delas. É possível afirmar, então, apenas que o acompanhamento à distância apresenta resultados semelhantes ao da literatura nacional e internacional, sendo uma alternativa viável no tratamento de crianças com alarme.

Pode-se também afirmar que outras variáveis não interferiram no resultado do tratamento para essa amostra, ou seja, que no trabalho realizado com 61 participantes, o alarme funcionou da mesma forma, independentemente de sexo, idade, frequência inicial de episódios e escolaridade dos pais. Porém, é sempre válido ressaltar que, em amostras diferentes ou maiores, tais resultados podem não se replicar.

Uma vez que as variáveis sexo, idade, frequência inicial de episódios e escolaridade dos pais não afetam a eficácia do alarme, aquele que aplica o tratamento com o aparelho pode realizá-lo independentemente dessas características da amostra. O clínico que utiliza esse método não precisa selecionar ou oferecer um tratamento diferenciado de acordo com nenhum desses fatores, o que facilita a difusão do tratamento.

A maior limitação deste trabalho está na questão metodológica da sobreposição das variáveis (1) tipo de acompanhamento e (2) problemas de comportamento, uma vez que os resultados indicam que ambas podem afetar o resultado do tratamento. Desta forma, não é possível ter clareza sobre o que afetou negativamente
Uma possibilidade futura seria repetir a investigação com a seleção e melhor distribuição dos participantes em cada grupo, de modo a garantir a sua homogeneidade pré-tratamento. Outra investigação de interesse seria não das variáveis moderadoras do tratamento com alarme, mas das mediadoras. Para isso, seria necessário investigar aspectos biológicos e comportamentais que dependeriam de análises mais sofisticadas e exames laboratoriais. Ou seja, o foco seria identificar quais mudanças - seja em termos fisiológicos, seja em condicionamento - que levam à obtenção de um controle que até o momento não havia sido instalado. Uma vez que ainda se sabe pouco sobre porque o alarme funciona, um estudo desse tipo poderia não esclarecer apenas o mecanismo de funcionamento do tratamento, mas também aspectos etiológicos da enurese que ainda permanecem obscuros.

\section{Referências}

Achenbach, T. M., \& Rescorla, L. (2001). Manual for the ASEBA school-age forms \& profiles. Burlington: ASEBA.

American Psychiatric Association. (2002). Manual diagnóstico e estatístico detranstornos mentais. Porto Alegre: Artmed.

Arantes, M. C. (2007). Problemas de comportamento e resultados do tratamento com alarme para enurese primária. Dissertação de mestrado não-publicada, Instituto de Psicologia, Universidade de São Paulo.

Butler, R. J. (2004). Childhood nocturnal enuresis: developing a conceptual framework. Clinical Psychology Review, 24 (8), 909-931.

Butler, R. J., Golding, J., Northstone, K., \& The ALSPAC Study Team. (2005). Nocturnal enuresis at 7.5 years old: prevalence and analysis of clinical signs. British Journal Urology International, 96 (3), 404-410.

Butler, R. J., \& Holland, P. (2000) The three systems: a conceptual way of understanding nocturnal enuresis. Scandinavian Journal of Urology and Nephrology, 34 (4), 270-277.

Butler, R. J., Holland. P., Gasson, S., Norfolk, S., Houghton, L., \& Penney, M. (2007). Exploring potential mechanisms in alarm treatment for primary nocturnal enuresis. Urology and Nephology, 41, 407-413.

Butler, R. J., Robinson, J. C., Holland, P., \& Doherty-Williams, D. (2004). An exploration of outcome criteria in nocturnal enuresis treatment. Scandinavian Journal of Urology and Nephrology, 38, 196-206.

Costa, N. J. D. (2005). A enurese noturna na adolescência e a intervenção comportamental em grupo $x$ individual com uso de aparelho nacional de alarme. Dissertação de mestrado não-publicada, Instituto de Psicologia, Universidade de São Paulo. 
Hjälmas, K., Arnold, T., Bower, W., Caione, P., Chiozza, L. M., von Gontard, H., et al. (2004). Nocturnal enuresis: an international evidence based management strategy. The Journal of Urology, 171, 2545-2561.

Houts, A. C. (1990). Guia dos pais para tratamento da enurese. São Paulo: USP.

Houts, A. C. (2003). Behavioral treatment for enuresis. In A. E. Kazdin \& J. R. Weisz (Orgs.), Evidence-based psychotherapies for children and adolescents (pp.389-406). New York: The Guilford Press.

Meyer, S. B., \& Vermes, J. S. (2001). Relação terapêutica. In B. Rangé (Org.), Psicoterapias cognitivo-comportamentais: um diálogo com a psiquiatria. Porto Alegre: Artmed.

Nevéus, T. (2008). Enuretic sleep: deep disturbed or just wet? Pediatric Nephology, 23 (8), 1201-1202.

Nevéus, T. (2009). Diagnosis and management of nocturnal enuresis. Current Opinion in Pediatrics, 21 (2), 199-202.

Nevéus, T., Eggert, P., Evans, V., Macedo, A., Rihing, S., Tekgül, S., et al. (2010). Evaluation and treatment for monosymptomatic enuresis: a standardization document from the International Children's Continence Society. The Journal of Urology, 183 (2), 441-447.
Nevéus, T., von Gontard, A., Hoebke, P., Hjälmas, K., Bauer, S., Bower, W., et al. (2006). The standardization of terminology of lower urinary tract function in children and adolescents: report from the standardisation Committee of the International Children's Continence Society. The Journal of Urology, 176 (1), 314-324.

Pereira, R. F. (2006). A enurese noturna na infância e na adolescência: intervenção em grupo e individual com uso de aparelho nacional de alarme. Dissertação de mestrado não-publicada, Instituto de Psicologia, Universidade de São Paulo.

Silva, R. P. (2004). Enurese noturna monossintomática: intervenção comportamental em grupos de pais e em grupos de crianças com aparelho nacional de alarme. Dissertação de mestrado não-publicada, Instituto de Psicologia, Universidade de São Paulo.

Silvares, E. F., Pereira, R. F., \& Arantes, M. C. (2007). Enurese. In R. M. Caminha \& M. G. Caminha. A prática cognitiva na infância (pp.254-271). São Paulo: Roca.

Recebido em: 30/4/2010

Versão final reapresentada em: 20/7/2011

Aprovado em: 9/8/2011

ANEXO

PROCEDIMENTOS

Tanto o contato pessoal como o telefônico implicaram um procedimento padronizado (Silvares, Pereira \& Arantes, 2007):

a) Observação ou levantamento das informações do registro escrito, questionamento de informações confusas, questionamento de anotações extras;

b) Verificação do seguimento das instruções gerais;

c) Interrogação dos motivos de não-seguimento das instruções, tais como acordar a criança no meio da noite para levá-la ao banheiro, não usar o alarme, não ajudar a criança a levantar quando o alarme é disparado;

d) Identificação e assinalamento dos sinais de progressos, tais como acordar mais rápido com o alarme, manchas menores, acionamento do alarme mais próximo da hora de acordar, diminuição do número de "molhadas" por noite, noites sem "molhadas", número de noites seguidas sem "molhadas";

e) Busca de soluções para as dificuldades e acompanhamento das estratégias propostas nas sessões subseqüentes. Exemplos: Se a criança desliga o alarme e volta a dormir, uma solução seria colocar o alarme em uma posição que dificultasse esse tipo de estratégia. Se a criança não consegue acordar com o alarme porque vai dormir muito tarde vendo filmes, uma possível solução seria disponibilizar uma forma de gravá-los;

f) Discussão buscando a identificação de fatores que, em cada caso individual, poderiam facilitar o seguimento dos procedimentos do alarme: presença de luz acesa, dormir cedo, dormir tranqüilo sem outras preocupações;

g) Acompanhamento de procedimentos adicionais, como o treino de autocontrole, treino de limpeza, técnicas de despertar e restrição de líquidos;

h) Discussão de dúvidas;

i) Avaliação da motivação e as expectativas tanto da criança quanto dos responsáveis e intervir, quando necessário;

j) Discussão de resistências ao uso do alarme apresentadas pelas crianças e manifestadas pelos pais;

k) Providências caso seja necessário realizar trocas no alarme. 
